\title{
STRUCTURE AND ELECTROCHEMICAL POTENTIAL SIMULATION FOR THE CATHODE MATERIAL $\mathrm{Li}_{1+\mathrm{x}} \mathrm{V}_{3} \mathrm{O}_{8}$
}

by

R. Benedek and M. Thackeray

Electrochemical Technology Program

Chemical Technology Division

Argonne National Laboratory

and

L. H. Yang

Condensed-Matter Physics Division

Lawrence Livermore National Laboratory

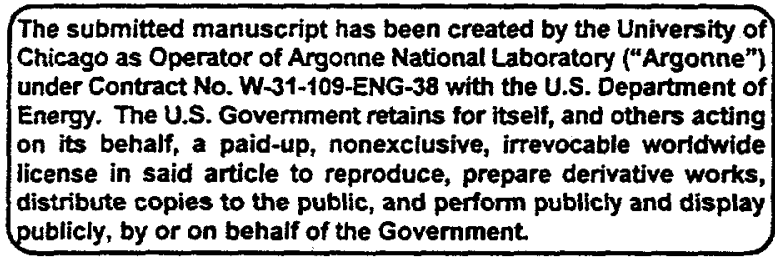

November, 1997

To be published in the Proceedings of the 1997 MRS Fall Meeting, December 1-5, 1997, Boston, MA. 


\section{DISCLAIMER}

This report was prepared as an account of work sponsored by an agency of the United States Government. Neither the United States Government nor any agency thereof, nor any of their employees, make any warranty, express or implied, or assumes any legal liability or responsibility for the accuracy, completeness, or usefuiness of any information, apparatus, product, or process disclosed, or represents that its use would not infringe privately owned rights. Reference herein to any specific commercial product, process, or service by trade name, trademark, manufacturer, or otherwise does not necessarily constitute or imply its endorsement, recommendation, or favoring by the United States Government or any agency thereof. The views and opinions of authors expressed herein do not necessarily state or reflect those of the United States Government or any agency thereof. 


\section{DISCLAIMER}

Portions of this document may be illegible in electronic image products. Images are produced from the best available original document. 


\title{
STRUCTLRE AND ELECTROCHEMICAL POTENTIAL SIMULATION FOR THE CATHODE MATERIAL $\mathrm{Li}_{1+x} \mathrm{~V}_{3} \mathrm{O}_{8}$
}

\author{
R. BENEDEK *, M. M. THACKERAY *, and L. H. YANG ** \\ *Chemical Technology Division, Argonne National Laboratory \\ **Condensed-Matter Physics Division, Lawrence Livermore National Laboratory
}

\begin{abstract}
The structure and electrochemical potential of monoclinic $\mathrm{Li}_{1+x} \mathrm{~V}_{3} \mathrm{O}_{8}$ were calculated within the local-density-functional-theory framework by use of plane-wave-pseudopotential methods. Special attention was given to the compositions $1+x=1.2$ and $1+x=4$, for which $x$-ray diffraction structure refinements are available. The calculated low-energy configuration for $1+x=4$ is consistent with the three Li sites identified in $x$-ray diffraction measurements and predicts the position of the unobserved $\mathrm{Li}$. The location of the tetrahedrally coordinated $\mathrm{Li}$ in the calculated low-energy configuration for $1+x=1.5$ is consistent with the structure measured by $\mathrm{x}$-ray diffraction for $\mathrm{Li}_{1.2} \mathrm{~V}_{3} \mathrm{O}_{8}$. Calculations were also performed for the two monoclinic phases at intermediate $\mathrm{Li}$ compositions, for which no structural information is available. Calculations at these compositions are based on hypothetical $\mathrm{Li}$ configurations suggested by the ordering of vacancy energies for $\mathrm{Li}_{4} \mathrm{~V}_{3} \mathrm{O}_{8}$ and tetrahedral site energies in $\mathrm{Li}_{1,5} \mathrm{~V}_{3} \mathrm{O}_{8}$. The internal energy curves for the two phases cross near $1+x=3$. Predicted electrochemical potential curves agree well with experiment.
\end{abstract}

\section{INTRODUCTION}

Transition-metal oxides that intercalate $\mathrm{Li}$ are under close scrutiny for their suitability as cathode materials in high-energy-density rechargeable batteries [1]. Among the desired properties of such components are high capacity, which is related to extensive intercalability, and long cycle life, which is related to structural stability upon $\mathrm{Li}$ insertion and extraction. A detailed characterization of atomic structure and phase stability as a function of $\mathrm{Li}$ insertion would be a valuable complement to electrochemical measurements for assessing the capabilities of a given candidate electrode material, as well as for suggesting strategies for improving the materials. It is frequently difficult, however, to obtain a comprehensive atomic structure characterization by experiment alone. Computational theory is a valuable additional tool for elucidating structural issues. As usual, different levels of computational theory may be considered, ranging from molecular dynamics [2] to semi-empirical tight-binding [3] to $a b$ initio methods. Implementation of local density functional theory (LDFT) is of considerable interest, since it is the most rigorous treatment currently feasible. Local density functional theory calculations for lithiated transition metal dioxides have been performed by others recently $[4,5]$.

In the present work, we apply plane-wave pseudopotential methods to the monoclinic trivanadate $\mathrm{Li}_{1+x} \mathrm{~V}_{3} \mathrm{O}_{3}$, a candidate Li-battery cathode [6], which intercalates between $1+x=1.2$ and $1+x=5$. The selection of this system was motivated, in part, by the availability of $x$-ray diffraction measurements [7] for the two monoclinic phases (which we refer to as $\alpha$ and $\beta$ ), whose most standard compositions are $1+x=1.2$ and $1+x=4$, which enable tests of the validity of our treatment. Compared to the dioxides $[4,5]$, the trivanadates are lower symmetry, larger unit cell systems, and therefore require greater computational effort. It is encouraging that local density functional theory appears to provide an accurate description of the trivanadate system. The structural information and and physical insights provided by these calculations are not readily obtained by other means. 


\section{METHOD}

Details of our plane-wave pseudopotential calculations are presented in a recent paper [8]. We employ Troullier-Martins pseudopotentials, a plane-wave basis set with 70 ry cutoff energy, and Gaussian-broadened Brillouin-zone sampling (with 16 special k-points in most cases). The Kohn-Sham equations for occupied and a few unoccupied orbitals are solved iteratively by a band-by-band conjugate gradient algorithm, stabilized by charge density mixing. The internal (reduced) atomic coordinates are relaxed to equilibrium under the guidance of the Hellmann-Feynman forces. The cell parameters (lattice constants) were held constant at the experimental values [7] for $\mathrm{Li}_{1.2} \mathrm{~V}_{3} \mathrm{O}_{8}\left(\mathrm{Li}_{4} \mathrm{~V}_{3} \mathrm{O}_{8}\right)$ in calculations for the low (high)-Li phase. The error introduced by the constant-lattice-parameter assumption is not expected to alter significantly the basic trends. For one composition, $\mathrm{LiV}_{3} \mathrm{O}_{8}$, lattice constants as well as internal coordinates were optimized self-consistently at constant energy cutoff. The calculated (experimental for $\mathrm{Li}_{1.2} \mathrm{~V}_{3} \mathrm{O}_{8}$ ) lattice constants (in a.u.) are $a=12.66(12.46), b=6.78(6.73), c=22.31(22.41)$. The level of agreement between theory and experiment is comparable to that typically achieved with LDFT. The energy difference between the calculation with experimental lattice constants and optimized lattice constants is approximately $0.1 \mathrm{eV}$ per formula unit. Zero-point energy for $\mathrm{Li}$ is neglected, since it is not expected to vary widely for different Li configurations at the same composition.

\section{RESULTS}

\section{Reference Systems}

The transition metal oxides under consideration are electronically strongly correlated systems that pose a severe test of LDFT. Although the trivanadate materials are known to be insulating, the energy bands calculated within LDFT show no energy gap at the Fermi level (except at the stoichiometric composition $x=0$, which does not occur in nature). Nevertheless, structural properties, which average over all valence electrons, are still reliably calculated within LDFT for many strongly correlated materials. Available atomic structure measurements for the reference compositions of the two monoclinic structures, $1+x=1.2$ and $1+x=4$, provide convenient tests of the LDFT framework for the trivanadates.

We discuss first the reference composition of the high- $\mathrm{Li}$ structure, $\mathrm{Li}_{4} \mathrm{~V}_{3} \mathrm{O}_{8}$. Anion and cation sublattices of this defect-rocksalt structure each have eight sites per formula unit, with one of the cation sites vacant. In principle, five different Li configurations come into consideration, since there are five non-vanadium cation sites on which the vacancy may reside. We label the configurations $438-1,438-2, \ldots, 438-5$, according to whether the vacant cation site is $\mathrm{Li}(1), \mathrm{Li}(2), \mathrm{Li}(3), \mathrm{S}_{\mathrm{oct}}(1)$ or $\mathrm{S}_{\text {oct }}(2)$, in the notation of de Picciotto et al. [7] The calculated energies (in parentheses, $\mathrm{eV}$ per formula unit) relative to the most stable are 438-5 (0.0), 438-4 (0.27), 438-3 (0.33), 438-2 (0.37), 438-1 (0.40).

Calculations have also been performed for the low-Li structure, whose standard composition is $\mathrm{Li}_{1,2}, \mathrm{~V}_{3} \mathrm{O}_{3}$. In the unit cell for this system with two formula units, the octahedrally coordinated $\operatorname{Li}(1)$ sites are fully occupied, and the tetrahedrally coordinated $\mathrm{Li}(2)$ sites have occupancy of 0.2 , according to XRD measurements [7]. Four other tetrahedral sites in this structure labeled $S_{\text {tet }}(1), \ldots, S_{\text {tet }}(4)$ are unoccupied. To verify that LDFT accurately describes the energetics of this structure, calculations were performed for a system with composition $\mathrm{Li}_{1.5} \mathrm{~V}_{3} \mathrm{O}_{8}$, in which both (octahedral) $\mathrm{Li}(1)$ sites are occupied but only one tetrahedrally coordinated $\mathrm{Li}$ site per unit cell is occupied. Five configurations were considered. in which the singly occupied site is either $\operatorname{Li}(2), \mathrm{S}_{\text {tet }}(1), \mathrm{S}_{\mathrm{tet}}(2), \mathrm{S}_{\mathrm{tet}}(3)$, or $S_{\text {ter }}(4)$. We label these configurations $1.538-1,1.538-2,1.538-3,1.538-4$ and $1.538-5$. 1.538 .1 is energetically favored over the others, whose relative energies are $1.538-1(0.0)$, $1.538-4(0.17) 1.538-2(0.29), 1.538-5(0.34), 1.538-3(0.62)$. 
The most stable of the calculated $\mathrm{Li}$ configurations for both $\mathrm{Li}_{1.5} \mathrm{~V}_{3} \mathrm{O}_{8}$ and $\mathrm{Li}_{4} \mathrm{~V}_{3} \mathrm{O}_{8}$ are found consistent with experiment. In the case of $\mathrm{Li}_{4} \mathrm{~V}_{3} \mathrm{O}_{8}$, only three of the four $\mathrm{Li}$ locations have been identified experimentally. These three sites are among the four that are occupied in the two lowest energy configurations 438-5 and 438-4. The configuration 4385 , however, has a substantially lower energy than 438-4, and our calculation therefore predicts that the fourth $\mathrm{Li}$ resides at the site $\mathrm{S}_{\text {oct }}(1)$. For $\mathrm{Li}_{1.5} \mathrm{~V}_{3} \mathrm{O}_{8}$ we find that the partially occupied site $\mathrm{Li}(2)$ is favored over the other possible tetrahedrally coordinated sites.

Figure 1 superposes theoretically predicted (circles) and experimentally determined (crosses) coordinates in a single $(010)$ layer of $\mathrm{Li}_{4} \mathrm{~V}_{3} \mathrm{O}_{8}$. The rms deviation between theoretical and experimental positions is about $0.15 \AA$. A qualitative feature of the structure is the curvature of the rows of oxygen, which reflects the stronger bonding to the vanadium than to lithium.

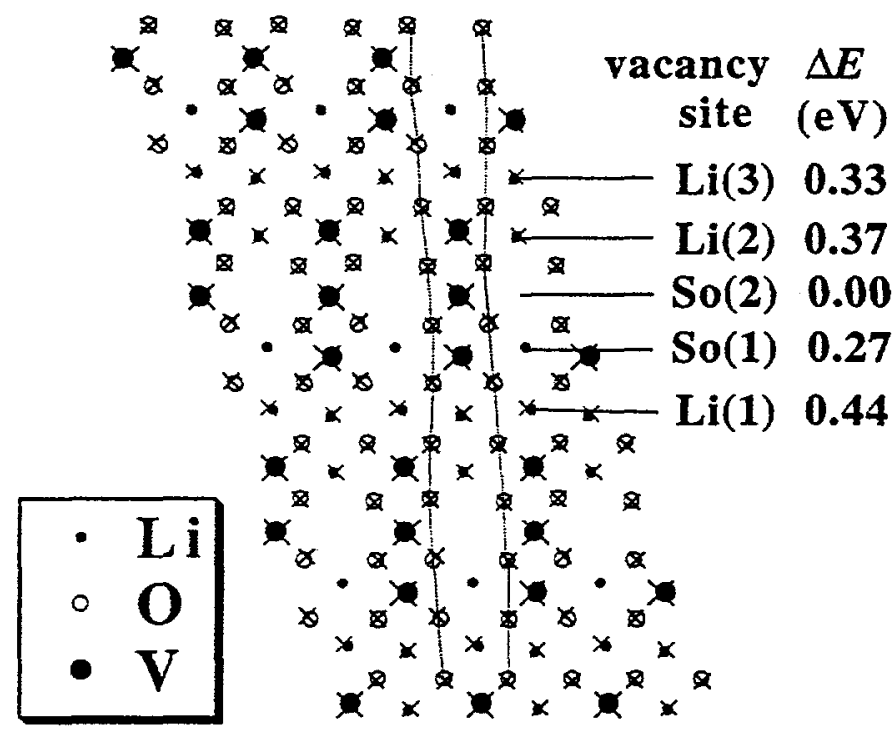

Fig. 1. Calculated (open and filled circles) and experimentally determined atomic positions [2] in a (010) layer of $\mathrm{Li}_{4} \mathrm{~V}_{3} \mathrm{O}_{3}$ in the 438-5 configuration, with a $\mathrm{Li}$ vacancy at $\mathrm{S}_{\text {oct }}(2)$. The calculated energies for other possible $\mathrm{Li}$ vacancy locations are indicated. Vertical rows of oxygen bend toward $\mathrm{V}$ trimers and away from $\mathrm{Li}$ dimers.

\section{Composition Dependence of Internal Energy}

A phase transformation occurs during lithiation between the low-lithium and the highlithium structures. Substantially different lattice parameters (7) for the two structures $\left(\mathrm{Li}_{1.2} \mathrm{~V}_{3} \mathrm{O}_{8}\right.$ and $\left.\mathrm{Li}_{4} \mathrm{~V}_{3} \mathrm{O}_{8}\right)$ suggest a first-order transition. To elucidate the relative stability of the two monoclinic structures, calculations were performed at intermediate $x$. In the absence of experimental atomic structure information except at the reference compositions, additional assumptions are required in order to proceed. Although evidence from both 
experiment and theory indicates that the reference compositions $\mathrm{Li}_{1.2} \mathrm{~V}_{3} \mathrm{O}_{8}$ and $\mathrm{Li}_{4} \mathrm{~V}_{3} \mathrm{O}_{8}$ are well ordered, configurational disorder is likely at intermediate compositions. We believe, however, that basic trends may be discerned from considerations of internal energy alone and disorder will be neglected.

The calculations for the reference system suggest that the energy cost of creating a $\mathrm{Li}$ vacancy in the hypothetical system $\mathrm{Li}_{5} \mathrm{~V}_{3} \mathrm{O}_{8}$ is in ascending order for a vacancy on sites $\mathrm{S}_{\text {oct }}(2), \mathrm{S}_{\text {oct }}(1), \mathrm{Li}(3), \mathrm{Li}(2)$, and $\mathrm{Li}(1)$. To delithiate the high-Li structure, we therefore depopulate sites in this order. Similarly we lithiate the low-Li structure by filling tetrahedrally coordinated sites in the order suggested by our results for $\mathrm{Li}_{1,5} \mathrm{~V}_{3} \mathrm{O}_{8}$. Although these prescriptions are somewhat arbitrary, similar qualitative features would most likely emerge from a more precise treatment.

Figure 2 shows the calculated energies per inserted Li relative to the hypothetical reference system $\mathrm{LiV}_{3} \mathrm{O}_{8}$ (i.e., $[E(1+x)-E(1)] / x$ ), as a function of $x$, for the low- and high$\mathrm{Li}$ structures. The high-Li structure shows an energy minimum at the standard composition $1+x=4$. We attribute the preference for this composition to the corresponding $\mathrm{V}^{4+}$ valence: local lattice relaxation stabilizes the single $d$-electron on vanadium, analogous to the JahnTeller effect.

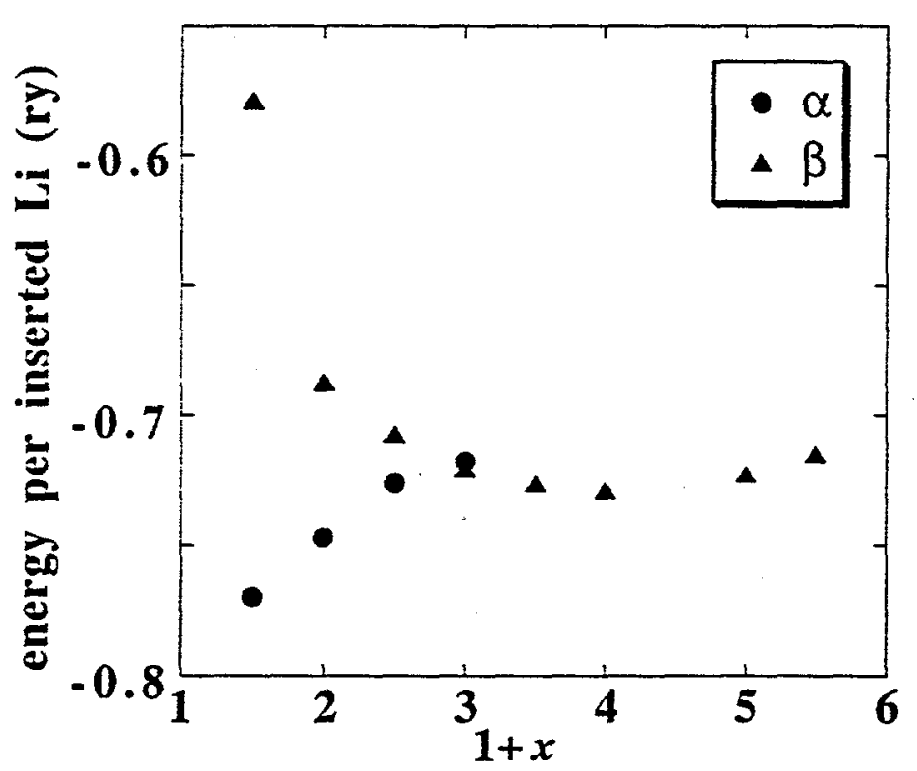

Fig.2. Calculated internal energy per inserted $\mathrm{Li}$, relative to $\mathrm{LiV}_{3} \mathrm{O}_{8}$, for the low-Li $(\alpha)$ and high- $\mathrm{Li}(\beta)$ structures.

Although free energy curves typically show positive curvature, the $x$-dependence of internal energy of the $\alpha$ (unlike the $\beta$ ) phase in Fig. 2 appears to have negative curvature. We note that the energies at certain compositions may be overestimated because of the selection of non-optimal Li-configurations. A more precise treatment would perhaps shift the crossing of the two curves, but probably only slightly.

The crossing of the low-Li and high-Li phase internal energy curves occurs in the vicinity of $1+x=3$. Since a line drawn between the circle at $1+x=1.5$ and the triangle at 
$1+x=5$ lies below the curves for $\alpha$ and $\beta$, equilibrium thermodynamics would predict twophase coexistence throughout. Owing to activation barriers, however, the nucleation of $\beta$ upon lithiation is delayed. The crossing of the internal energy curves (at $1+x=3$ ) occurs close to the composition at which a two-phase (see the voltage plateau in Fig. 3) process is thought to commence during electrochemical lithiation $[6,9]$. This proximity may be accidental, however, if kinetics is the controlling factor.

Our calculations provide clues regarding transformation paths. As $\mathrm{Li}$ is removed from the high-Li structure, features characteristic of the low-Li structure start to develop, while the exclusively octahedral $\mathrm{Li}$ coordination characteristic of the high-Li structure remains intact. Specifically, the curvature of the rows of oxygen becomes increasingly pronounced, as does the curvation of the vanadium trimers. The effect of the increased curvatures is to open corridors for $\mathrm{Li}$ movement, thereby lowering activation energies for transforming to the preferred low-Li structure at depleted $\mathrm{Li}$ compositions.

\section{Electrochemical Potential}

We have analyzed the calculated energies for the two trivanadate phases to predict electrochemical potentials, relative to a pure- $\mathrm{Li}$ anode. The chemical potential of $\mathrm{Li}$ in the oxide is obtained from the difference between total energies for cells in which the lithiation differs by the increment $\Delta x=0.5$ or 1.0. The calculated electrochemical potentials are shown in Fig. 3.

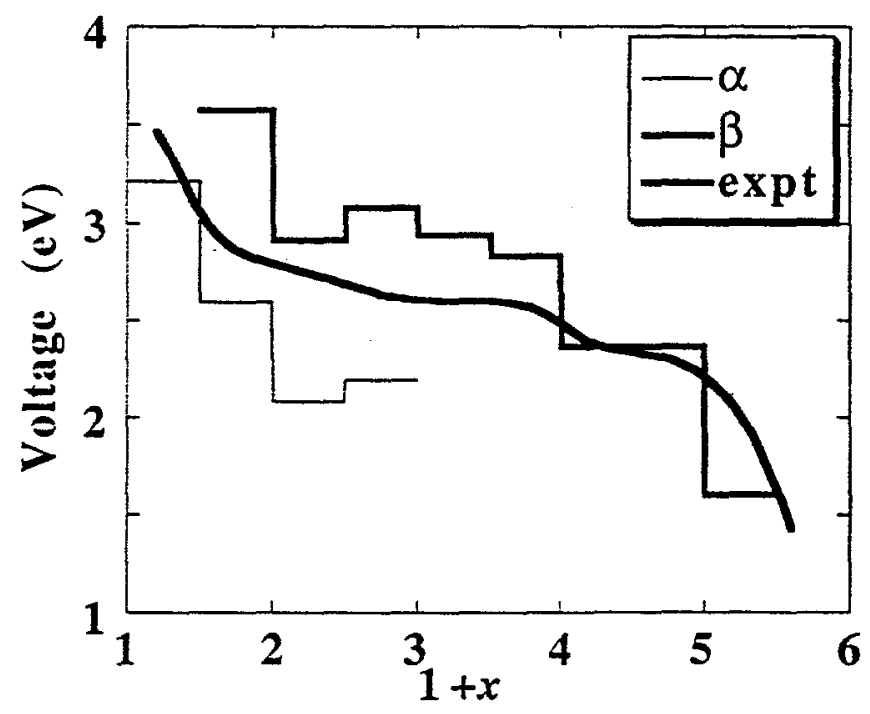

Fig. 3. Calculated electrochemical potential for $\mathrm{Li}_{1+x} \mathrm{~V}_{3} \mathrm{O}_{8}$ versus lithium filling, for the $\alpha$ (low-Li) and $\beta$ (high-Li) phases. Voltage drops sharply at $1+x=5$, since all vacancies in the defect rocksalt structure are filled.

Also shown for comparison is an experimental cell voltage curve at low current [9]. It is encouraging that the $a b$ initio calculations, with no adjustable parameters, are relatively close to experiment. To make a more detailed interpretation requires a hypothesis regarding the structure in the region between the standard compositions $(1+x=1.2,4)$. The most straightforward interpretation of the experimental electrochemical potential curve is that 
pure $\alpha$ persists up to $1+x=3$, where a two phase regime begins. In this case, the $\alpha$-phase predicted electrochemical potential at $1+x>2$ is underestimated. The fixed lattice-parameter approximation, the non-optimal selection of $\mathrm{Li}$ configurations, and the neglect of entropic contributions to the free energy may all contribute to this discrepancy. Incidentally, the sharp drop in voltage above $1+x=5$ is attributed to the lack of additional vacancies for $\mathrm{Li}$ to fill in the defect rocksalt structure. Our calculation for $1+x=5.5$ treats a single $\mathrm{Li}$ in an interstitial site, which results in a lower voltage than those that correspond to filling vacancies.

\section{CONCLUSION}

$A b$ initio LDFT calculations for the trivanadate compositions $\mathrm{Li}_{1.5} \mathrm{~V}_{3} \mathrm{O}_{8}$ and $\mathrm{Li}_{4} \mathrm{~V}_{3} \mathrm{O}_{8}$ yield structural coordinates in excellent agreement with experiment, as well as predictions of $\mathrm{Li}$ coordinates missing from the XRD refinements. Internal energy curves for the lowand high-Li phases cross in the vicinity of $x=3$. Electrochemical potential simulations (Fig. 3 ) for the $\alpha(\beta)$ phase are in close accord with experiment, particularly in the interval $1+x<2(1+x>2)$, but the detailed interpretation is obscured by the lack of experimental structural information in the region between the standard compositions $(1+x=1.2,4)$.

\section{ACKNOWLEDGMIENTS}

This work was supported at ANL by the U. S. Department of Energy, Office of Basic Energy Sciences. L.Y. is supported at LLNL by the U. S. Department of Energy under contract no. W-7405-ENG-48.

\section{REFERENCES}

1. P. G. Bruce, Chem. Commun. 19, 1817 (1997).

2. R. T. Cygan, H. R. Westrich, and D. H. Doughty, Mat. Res. Soc. Symp. Proc. 393, $113(1995)$.

3. A. Stashans, S. Lunell, R. Bergstroem, A. Hagfeldt, and S.-E. Lindquist, Phys. Rev. B53, 159 (1996).

4. M. K. Aydinol, A. F. Kohan, G. Ceder, K. Cho, and J. Joannopoulos, Phys. Rev. B56, 1354 (1997).

5. C. Wolverton and A. Zunger, Phys. Rev. B., in press (1997).

6. K. West, B Zachau-Christiansen, S. Skaarup, Y. Saidi, J. Barker, I. I. Olsen, R. Pynenburg, and R. Koksbang, J. Electrochem. Soc. 143, 820 (1996).

7. L. A. de Picciotto, K. T. Adendorff, D. C. Liles, and M. M. Thackeray, Solid State Ionics 62, 297 (1993).

8. R. Benedek, M. M. Thackeray, and L. H. Yang, Phys. Rev. B56, 10707 (1997).

9. V. Battaglia, A. Jansen, and A. Kahaian, unpublished. 